\title{
A digital replica of male compression underwear
}

\author{
Zhe Cheng ${ }^{1,2} \oplus$, Victor Kuzmichev ${ }^{2,3} \oplus$ and \\ Dominique Adolphe ${ }^{4}$
}

\begin{abstract}
With the development of society and increasing demands, the design of male underwear should conform to human ergonomics. However, some special delicate aspects related to morphological features of the male body need to use computer technologies for generating a digital replica of real bodies, with textile materials instead of real objects from the perspective of satisfying increasing needs. This article aims to apply three-dimensional (3D) simulation technology in compression clothes design. We have proposed the method of material precision simulation, body avatar establishment, and virtual underwear design. In this study, multiple 3D software to establish avatars of male bodies with different morphological features are used first. Secondly, the conversion of the Kawabata Evaluation System for Fabrics results in a digital library of knitted materials to be explored, which are mainly based on elastic characteristics and compression performances. Then, we conducted a series of try-on tests with average size avatars, which are created using 3D scanning technology, and evaluated the pressure and comfort using a new method we recommended. Finally, the results of the virtual and actual tests are compared. All digital replicas achieve the same results as the real ones. This study can promote the application of 3D simulation technology in male compression underwear design by operating a digital replica instead of real objects.
\end{abstract}

\section{Keywords}

computer-aided design, virtual reality, digital replica, textile material, underwear

With the development of science and industrial 4.0 (I4) digital technology, the apparel industry needs to combine traditional production services with the new manufacturing process by means of innovation and computerization. From two-dimensional (2D) pattern drawing to the wide application of three-dimensional (3D) technologies, which starts from body scanning and end with try-on simulation, the apparel industry has developed in recent years. ${ }^{1-3}$ The developments convert apparel manufacturing from traditional 2D design to a new type of process. ${ }^{4,5}$ Therefore, apparel should be developed in terms of design and engineering by using digital technologies. ${ }^{6}$

Under this trend, many explorations have been done to apply virtual technologies to apparel design. Some simulation experiments target $2 \mathrm{D} \rightarrow 3 \mathrm{D}$ or $3 \mathrm{D} \rightarrow 2 \mathrm{D}$ approaches for different apparel kinds and different body morphologies. ${ }^{7-9}$ Some experiments focused on the simulation of the textile materials, styles, and appearances or the calculation of apparel size, for a specific group of people. ${ }^{10-13}$ Some scholars studied the wearing performance between the virtual apparel and the human body. ${ }^{14,15}$ Moreover, most experiments only used a limited number of virtual avatars or scanatars (scanned testees), which could not be considered as good digital replicas of the human body. ${ }^{16,17}$ The drawback is that the simulation does not involve different apparel sizes and types according to the features of human body morphologies. ${ }^{18}$ In general, due to the

\footnotetext{
'Wuhan Textile University, China

${ }^{2}$ Wuhan Textile and Clothing Digital Engineering Technology Research Center, Wuhan, China

${ }^{3}$ Ivanovo State Polytechnical University, Russian Federation

${ }^{4}$ Universite de Haute Alsace, France

\section{Corresponding author:}

Victor Kuzmichev, Ivanovo State Polytechnical University, Ivanovo 153000, Russian Federation.

Email:wkd37@list.ru
} 
absence of comparison of real objects and virtual analogs, it is impossible to predict the real effect of $3 \mathrm{D}$ design.

The evaluation of apparel comfort, fit, and virtual performance are hot research topic now. ${ }^{19}$ Most researchers cannot control and change the properties of virtual textile materials or the dimensions of body morphology under try-on simulation, so it is difficult to control the fit of e-apparel. ${ }^{20}$ Some designers only adjust the 2D apparel pattern block according to the visual effect displayed in the 3D simulation system, which is usually used in loose clothing design. ${ }^{21,22}$ Usually, the virtual textile materials presented a limited amount of own properties in the shaping of loose-fitting apparel, but for tight-fitting apparel, the more significant material properties and pressure comfort should be taken into account. ${ }^{23}$ The structural design and the perception of the human body vary with different materials. ${ }^{24}$ Therefore, currently it is impossible to evaluate apparel in virtual reality (VR) in terms of sensory analysis. For better comparison of the virtual and real objects, as well as comprehensive prediction of the real product, it is necessary to combine theory with practice for verification and to make long-term, comprehensive research, application evaluation, etc. ${ }^{25,26}$

Furthermore, there are many 2D and 3D computeraided design (CAD) software programs in the apparel industry, which has advantages and disadvantages. Their applications show good effects in pattern drawing two or three dimensions, ${ }^{27,28}$ but the instruments are not good at generating human body avatars with complex morphology and a soft structure, the virtualization of the perception (such as compression sensitivity of human soft tissue), the performance of textile materials, etc. As usual, the performance simulation of real system "avatar-apparel" cannot be completed with only one software. Therefore, to do the simulation in VR with real experimental data, it is necessary to integrate various software programs and create new databases. Finally, the system "avatar-apparel" as a complex digital replica of the real one can be obtained by joining three digital objects, namely the human body, textile materials, and apparel.

\section{Objectives and aims}

The design of compression underwear is a multidisciplinary study that involves knowledge from anthropometry, medicine, textile science, the pattern design, and image analysis based on systematic approaches. The results of the design directly affect the comfortable feeling and health. At present, only a few researches are focusing on this kind of apparel and only concentrating on the selection of textile materials, the pressure experiments, or the development of the avatars. ${ }^{29-31}$
Nevertheless, in our methodology of design and simulations, we took into account the real data about human body morphology, textile materials, comfort evaluation, and physiological performance. This study demonstrated the original experimental data and the method of its adjusting in VR to achieve the same results as the reality by using two digital replicas of the textile materials and the compression underwear to satisfy the wearing comfort of body under the influence of various factors (compression pressure, material deformation, human body reshaping, etc.). The evaluation criteria were designed to compare the two kinds of results - real and virtual.

In order to describe the accuracy of underwear simulation, it is necessary to evaluate the "fit" - defined as the relationship between the outline shape of apparel and the body morphology, which is always judged in terms of the apparel appearance and the sensory analysis evaluation. ${ }^{32-34}$ A large number of scholars have explored the apparel fit from different points of view confirmation between the apparel style and the contours of the body, the presence or absence of wrinkles, the configuration of structural and bottom lines, values of ease allowance, the behavior of materials, assessment of health, or the body feelings. ${ }^{35,36}$ For elastic compression apparel, the ease allowance, location of structural lines, and pressure feeling are often used to measure the comfort/fit. In this study, the underwear fit is analyzed by the subjective and objective evaluation of the testees, pressure measuring, calculation of the ease allowance, and structure design.

In this paper, a technical method for obtaining the same results as the actual samples by simulation is proposed. The proposed method verifies the following items: (a) minimize the differences between the results obtained after VR and real testing; (b) improve material simulation; (c) build up the digital replicas of human bodies, knitted materials, and underwear that should conform to the real prototypes; (d) measure the pressure between the avatar and the virtual materials and its deformation; (e) build up a criterion to evaluate the pressure in virtual system "avatar-underwear" as the indicator of comfort. Using the mentioned items, we proved the reliability of our method of the design process digitalization, the possibility of contemporary $3 \mathrm{D}$ software being applied in a completely new area, and the accuracy of evaluation. In summary, we improved the efficiency of compression underwear design by direct evaluation of the accuracy and fit in VR.

In our methodology, we first describe the entire study process and the software used, as well as the key steps, in the first section. We present the preliminary experimental details (basic database), including the anthropometric measurements, 3D rigid scanatars, and Kawabata Evaluation System (KES) tests of materials 
in the second section. In the third section, we describe the simulation methods by extracting the multiple body measurements to build the avatars with the various sizes and put them into CLO software; the mechanical-physical properties of 14 materials and the interaction between the materials and the different parts of the human body are also simulated. In the fourth section, we describe the establishment and adjustment of the digital replicas based on real indexes and dimensions of the materials and the bodies, and we present the accuracy of the proposed digital replica of the male body by comparison and evaluation of the real and virtual results obtained, which gave the 3D underwear adequate material properties. Finally, a discussion of experimental accuracy and application prospects is conducted, along with analysis of the study constraints and proposed possible further work.

\section{Methodology}

In our study, we investigated two systems: the real system "body-underwear" and the virtual system "avatar-underwear" as a digital replica. The experiment consists of four phases: (a) the development of a new basic database to improve the possibilities of the existing software; (b) the application of the new database to generate three digital replicas; (c) the evaluation of all digital replicas generated by comparison with real prototypes; (d) results. Experimental studies were carried out according to the flowchart shown in Figure 1.

This process was designed to simulate the behavior of the delicate morphological features of males under compression and reshaping by stretching knitted materials. The method consists of four main components.

- The development of rigid avatars, modification of the morphological features, and transformation of deformed avatars using multiple 3D software, such as MakeHuman and 3ds Max (Steps A1 and A2).

- Development of the digital replica of knitted materials after testing the properties by the KES, evaluation of the compression ability, and its conversion into virtual data by using special equations with CLO (Steps B1-3).

- Optimization of the underwear pattern block (Steps C1-3).

- Virtual try-on to demonstrate the applications and possibilities of the new database (Steps D1 and D2). This method requires additional manual steps to simulate the soft tissues of the avatars after being lifted.

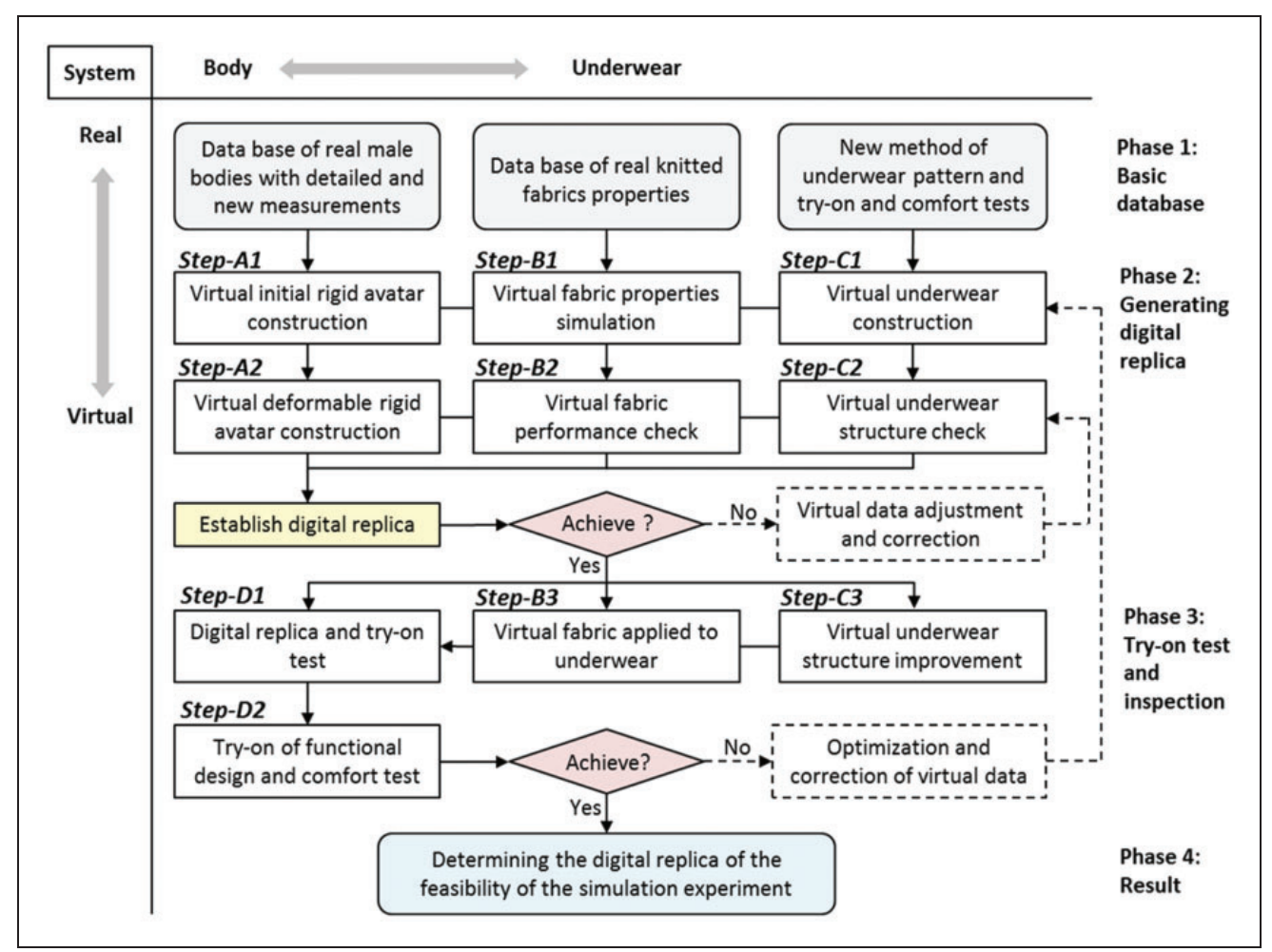

Figure I. Flowchart of the design process of how to use the digital replica of all elements of the system "avatar-underwear." 
Table I. The steps and software used in the study

\begin{tabular}{|c|c|c|c|}
\hline No. & Software & Advantages and disadvantages & Application in our study (steps) \\
\hline I & MakeHuman I.I & $\begin{array}{l}\text { It can quickly create avatars with different } \\
\text { characteristics (race, gender, size, posture, } \\
\text { etc.) } \\
\text { Disadvantage: mainly for avatar construction, } \\
\text { cannot be used for try-on, evaluation, or to } \\
\text { measure the dimensions of any avatars }\end{array}$ & $\begin{array}{l}\text { Step AI - to set the initial male avatar } \\
\text { Step A2 - to add the "male genital" } \\
\text { component, then to import into } 3 \mathrm{ds} \\
\text { Max }\end{array}$ \\
\hline 2 & $3 \mathrm{ds}$ Max 2015 & $\begin{array}{l}\text { It can modify and shift the details of virtual } \\
\text { apparel accurately } \\
\text { Disadvantage: not based on the apparel } \\
\text { simulation and evaluation, does not have } \\
\text { objective reference values for evaluation of } \\
\text { the apparel fit }\end{array}$ & $\begin{array}{l}\text { Step } \mathrm{A} 2 \text { - to modify the avatar by } \\
\text { producing the push-up effect of soft } \\
\text { tissue, then import into CLO } 4.1\end{array}$ \\
\hline 3 & Richpeace CAD V8 & $\begin{array}{l}\text { It can draw and adjust the pattern block. } \\
\text { Disadvantage: cannot be expressed in 3D } \\
\text { method, and the adjustment is too } \\
\text { complicated }\end{array}$ & $\begin{array}{l}\text { Step } \mathrm{Cl} \text { - drawing of underwear pat- } \\
\text { terns } \\
\text { Steps } \mathrm{C} 2, \mathrm{C} 3 \text { - optimization of pattern } \\
\text { blocks, drawing the exact pattern } \\
\text { and importing into CLO } 4.1\end{array}$ \\
\hline 4 & CLO 4.I & $\begin{array}{l}\text { The 2D pattern is made synchronously with } \\
\text { 3D simulation, the pattern block and 3D } \\
\text { apparel can be flexibly adjusted; can meas- } \\
\text { ure sizes, pressure, deformation; excellent } \\
\text { simulation performance, flexible modifica- } \\
\text { tion of the avatar postures in static and } \\
\text { dynamic; wide range of simulated proper- } \\
\text { ties/value; very good simulation of common } \\
\text { textile materials and apparel (coat, dress } \\
\text { etc.) from commonly used materials } \\
\text { (cotton, silk, etc.) } \\
\text { Disadvantage: the avatar looks not like a real } \\
\text { human in some details; the software oper- } \\
\text { ates only with its own virtual materials. If } \\
\text { the material data is tested by other devices, } \\
\text { it needs to be re-examined }\end{array}$ & $\begin{array}{l}\text { Steps } \mathrm{AI}, \mathrm{A} 2 \text { - checking an avatar for } \\
\text { try-on } \\
\text { Steps } \mathrm{BI}-3 \text { - simulation of performance } \\
\text { of knitted material, measuring the } \\
\text { virtual pressure values, and evalu- } \\
\text { ation of accuracy and reliability } \\
\text { Steps } \mathrm{Cl}-3 \text { - checking of pattern block } \\
\text { and building virtual underwear for } \\
\text { try-on } \\
\text { Steps DI and D2 - simulation under- } \\
\text { wear in various sizes for different } \\
\text { bodies and evaluation of the design } \\
\text { and wearing comfort }\end{array}$ \\
\hline
\end{tabular}

3D: three-dimensional; 2D: two-dimensional.

Table 1 shows the details of all the mentioned components.

The main definitions of this study are as follows.

- The digital replica is a copy of the real object with the similar features that can simulate different real situations in VR and produce similar reactions to those of the real object. ${ }^{38-40}$ Three types of digital replica will predict the relationship between the three objects to improve the efficiency of underwear design instead of the male body, knitted materials, and compression underwear.

- The rigid avatar is a 3D model, which has the same dimensions as the real human body, created in VR by using 3D software.

- The deformed avatar is a 3D geometrical model that can be transformed from the rigid avatar and has possibilities to adjust the dimensions and morphology in accordance with the textile materials stretching and soft tissue compression and perform displacement or modification of the 3D model components according to the adjustable range of the real male body.

- Basic underwear is the basic style design to cover the lower part of the human torso.

- Functional underwear is the specific style with special functions designed to produce the lifting effect of genitalia and buttocks and to deform the avatar by a tightening effect after textile material stretching.

- The push-up/lifting effect is the result of human body deformation due to the interaction between the knitted materials stretching and soft tissues under the generated contact pressure and complex force transmission, which involves material stretching and soft tissue compression. 
The push-up effect cannot be designed in the underwear pattern block accurately without the evaluation of real samples. So, virtual detection (Steps A2 and D2) can be designed in parallel to conclude the try-on evaluation. The approach will help to evaluate the effectiveness of underwear functions and to verify the push-up and compression effects in VR. However, due to limitations of the MakeHuman software, it is impossible to simulate the push-up by using the initial rigid avatar. Therefore, it is necessary to build a variety of deformed avatars to test the influence of the underwear structure and the deformation of knitted materials for more detailed human bodies in Step A2.

So, the virtual experiments included body modeling, the modification of underwear structure, the simulation of knitted materials properties, the evaluation of underwear comfort, etc. When the results are not satisfactory, the simulation process will be performed again.

\section{Preliminary experimental details}

\section{Information collection}

Previously, the main problems that arise during underwear wearing have been identified through consumer preference studies involving nearly 800 men in Russia, France, China, and Bangladesh. According to consumers' demands and problems, the following experiments have been completed to parameterize the new database in terms of the CAD application: (a) information about the morphology of male bodies and their soft tissue sensitivity; (b) new classifications of the lower part of male bodies adapting to an underwear structure; (c) analysis of the influence of knitted material properties on compression comfort; (d) new methods of underwear pattern block making by using additional body measurements. After all the mentioned experiments, completely new databases as CAD information support have been obtained to improve the virtual simulation and to obtain digital realistic looking replicas of male bodies, textile materials, and underwear.

\section{Male body morphology}

One-hundred-and-fifteen young males (aged 18-28) with ordinary body shapes and who are not overweight were measured by a Vitus Smart XXL 3D body scanner following the standard DIN EN ISO 20685. Their body measurements were determined by Anthroscan 3D image processing software. The main measurements were as follows: height $174.4 \pm 9.3 \mathrm{~cm}$; waist girth (WG) $76.2 \pm 6.8 \mathrm{~cm}$; hip girth (HG) $94.2 \pm 5.5 \mathrm{~cm}$; crotch height $77.5 \pm 5.3 \mathrm{~cm}$.

All bodies are divided into several groups to build up a more exact database of the scanatars generated. The sagittal sections of the scanatars have been taken to develop new classification sizes and overlapping at the same natural waist height. Table 2 includes three types of male torsos in accordance with our exploration: small, middle, and large.

Eighteen additional body measurements for the description of male lower torsos and underwear pattern block design are proposed, including the significant variables to represent the soft tissue of the front (genitalia) and buttocks and its lifting mobility. This special database can be used to simulate the soft tissues in Steps A1 and A2 and the underwear functional effects in Steps C1-C3 (MakeHuman, CLO, 3ds Max).

\section{Knitted material properties}

Fourteen types of knitted materials (T1-T14) that are popular in men's underwear are studied in terms of fiber and structure. Most knitted materials were made of Lenzing Modal (43-93\%), viscose (45-60\%),

Table 2. The average sizes of the measured male torso, $\mathrm{cm}$

\begin{tabular}{|c|c|c|c|c|c|c|c|c|c|c|c|c|}
\hline Girth & \multicolumn{4}{|c|}{ Small S } & \multicolumn{4}{|c|}{ Middle M } & \multicolumn{4}{|c|}{ Large L } \\
\hline Hip girth & \multicolumn{4}{|c|}{$<92$} & \multicolumn{4}{|c|}{$92-98$} & \multicolumn{4}{|c|}{$>98$} \\
\hline Avg. waist girth & 70 & 73.5 & 84 & 91 & 74 & 77.5 & 88 & 99 & 77 & 80.5 & 91 & 102 \\
\hline Sagittal sections & & & & & & & & & & & & \\
\hline
\end{tabular}


combed cotton (20-65\%), or polyethylene (90-97\%), and mixed with spandex (3-35\%). The structures are weft knit - single and double sides of plain knit (jersey), single and double sides of rib knit; warp knit - interlock knit, pique knit. Four knitted materials were relatively thick $(0.9-1.2 \mathrm{~mm}) ; 10$ materials were thin $(0.4-0.8 \mathrm{~mm})$. Nine knitted materials have weight of $110-180 \mathrm{~g} / \mathrm{m}^{2}$, and five have weight of $200-360 \mathrm{~g} / \mathrm{m}^{2}$. The average shrinkages in the warp and weft are $2.4-5.5 \%$. So, the chosen knitted materials represent the full range of those used for underwear production.

Figure 2 shows the surface of several materials in smooth and wrinkled positions and their ability to shape.

All knitted materials are tested by the Kawabata Evaluation System for Fabrics (KES-F) to evaluate their parameters of physical and mechanical properties. We choose the KES-F because this instrument allows testing the behavior of textile materials more accurately and more closely to real wearing conditions than the Fabric Assurance by Simple Testing (FAST) system. ${ }^{41}$ We measured 15 material parameters of tension $(R T$, $E M T \ldots)$, shear $(G, 2 H G \ldots)$, compressibility ( $L C$, $R C \ldots)$, thickness, and friction (MIU ...). After complex analysis, the following parameters are extracted for the simulation:

- resiliences of tensile $R T$ are $20.17-78.50 \%$ (100\% means that the resilience is good);

- elongations of tensile EMT under high load of $500 \mathrm{cN} / \mathrm{cm}$ are $15.77-195.06 \%$ (the higher the value, the better the tensility);

- rigidity/stiffness values of shear $G$ are $0.03-0.91 \mathrm{gf} /$ $\mathrm{cm}^{\circ}$ (the higher value, the harder the shearing).

Since the load $F$ of the EMT test is too large $(500 \mathrm{gf} / \mathrm{cm})$, the smaller load $F$ is chosen in the experiment; the large load $F$ will increase the elongation of the textile materials, and the excessive elongation (much tighter to the human body) is not suitable for underwear design. When the fabric reaches a certain elongation (which we call the maximum elongation $\left.E_{\max }\right)$, the acceptable comfort pressure critical value of the male body can be determined, and the average
$F\left(E_{\max }\right)$ is extracted when the fabric reaches $E_{\max }$ of $81.24 \mathrm{cn} / \mathrm{cm}$.

Moreover, through the pressure comfort tests on male bodies, it was found that different knitted materials can produce different compression, so they should be separated for further simulation in VR. We propose the index of compression performance $(C P, \mathrm{kPa} / \%)$, which indicates the pressure value that the material can produce under elongation of $1 \%$. The calculation is as follows

$$
C P=P_{\max } / E_{\max }
$$

where $E_{\max }$ is the maximum elongation of knitted material under which the material produces the maximum acceptable pressure, $\% ; P_{\max }$ is the maximum pressure value tested on the body, $\mathrm{kPa}$.

According to real tests, the average $C P$ for tested materials is near $0.091 \mathrm{kPa}$. We divided all knitted materials into four levels in accordance with their $C P$ index:

- first level with the weakest $C P$ of less than $0.050 \mathrm{kPa} / \%$;

- second level with $C P$ below average, $0.050 \leq C P<0.100 \mathrm{kPa} / \%$;

- third level with $C P$ above average, $0.100 \leq C P<0.150 \mathrm{kPa} / \%$;

- fourth level with $C P$ more than $0.150 \mathrm{kPa} / \%$.

\section{Sensitiveness of soft tissues}

Before the simulation, some data about the maximum pressure and elongation are obtained on the real male body, as shown in Figure 3.

These data from Figure 3 are equivalent to the ease allowance $E_{\max }$ and the appropriate pressure $P_{\max }$, which can be used for the numerical reference of the simulation. The data were obtained through sensory analysis of a real testee when the pressure was measured over the girth of the human body by an objective method (Tekscan Inc. USA, Flexi Force A201-L sensor, sensing area $70 \mathrm{~mm}^{2}$, error smaller than (a)

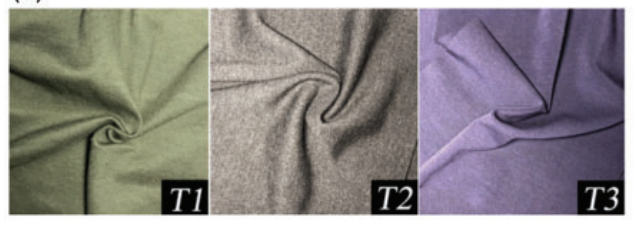

(b)

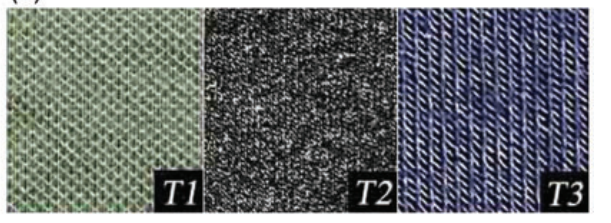

Figure 2. Knitted material samples (a) and twice enlarged images (b). 
$\pm 3 \%$, and LabVIEW system $9.6 \mathrm{kHz}$, value recorded at 10 times/s). ${ }^{42-44}$ According to the different characteristics of the human body, the number of measurement points on each part is more than 10, including bones and fat tissue, the pressure is measured three times at each point, and the average values are calculated in each experiment. Although knitted materials are elastic, they could not always touch the skin due to the complex geometry of the body girth. In the convex parts, the skin and the material have good contact, but in the concave parts, the lack of contact leads to absent pressure. ${ }^{45}$ The average values of $P_{\max }$ and $E_{\max }$ are $1.57 \pm 0.55 \mathrm{kPa}$ and $-20.5 \pm 4.2 \%$, respectively. The pressure measured near the anterior superior iliac spine at the waistband is $3.18-5.14 \mathrm{kPa}$.

It is necessary to establish an indicator of the sensory evaluation that will reflect the real feelings under pressure to perform the same evaluation only by numerical values. We established five grades to indicate the pressure under the underwear, as shown in Table 3.

The evaluation scale is calculated from the average measurements at different girths. The full pressure range is suitable for the human body. This range is justified in some related studies. ${ }^{46-51}$ The evaluation scale evaluates the pressure from weak to strong.

- Level-1 (the weakest pressure) - not tight, does not produce support for soft tissue, very light, suitable

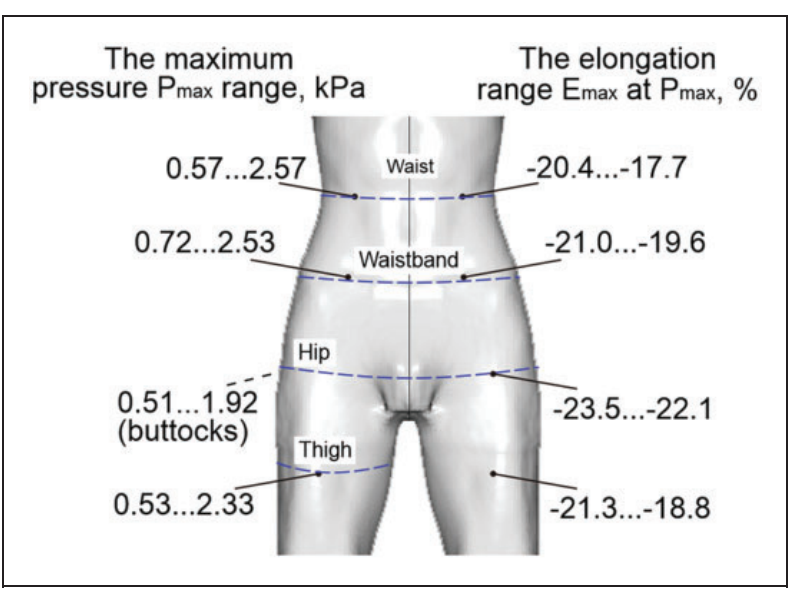

Figure 3. Areas of the male lower torso with different sensitiveness. for daily or sleeping underwear; the reason for application is mainly due to the weak compression properties of the materials.

- Level-2 (weak pressure) - not tight, suitable for daily or sleeping underwear.

- Level-3 (median pressure) - a little tight, suitable for daily underwear.

- Level-4 (strong pressure) - tight and good support, suitable for daily or sports underwear.

- Level-5 (the strongest pressure) - very tight, suitable for sports and functional underwear.

\section{Generating the digital replica of system "avatar-underwear"}

\section{Digital replicas of male bodies}

MakeHuman can set the initial rigid avatars of the 3D male body with the necessary measurements by adding the "male genitalia" plug-in. Then, the initial rigid avatar can be improved by 3ds Max by modifying some parts (as after lifting). We provided three possible ways to build the avatar and to form the virtual systems:

- system "initial rigid avatar A1 + basic underwear";

- system "initial rigid avatar A1 + functional underwear";

- system "deformed avatar A2 + functional underwear."

The initial rigid avatar A1 has the genitalia part in the natural falling state. We have adjusted the average volume of genitalia approximately for Asians males (near $45 \mathrm{~cm}^{3}$ ). The deformed avatar is obtained from the initial rigid avatar by displacement of the front and back "soft tissue" components according to the real experimental data. We changed the height and state of the "soft tissue" to an upward state like wearing push-up underwear.

\section{Initial rigid avatars with body measurements}

During Step A1, 12 avatars named "initial rigid avatar A1" were created to present the real bodies with the measurements from Table 1 after adjustment of waist $W_{G}$, hip $H_{G}$, and thigh $T_{G}$ girths, genitalia and buttocks

Table 3. The evaluation scale of virtual pressure

\begin{tabular}{llllll}
\hline Grades & 1 & 2 & 3 & 4 & 5 \\
\hline $\begin{array}{l}\text { Colorful pressure } \\
\text { comfort scale }\end{array}$ & & & & & \\
Pressure, $\mathrm{kPa}$ & 0.5 & 1.0 & 1.5 & 2.0 & 2.5 \\
\hline
\end{tabular}


bulge and height, etc. The algorithm of initial rigid avatar A1 is built up, including the following steps:

- creation of the basic initial rigid avatar A1 and adding the "genitalia" by using MakeHuman;

- adjusting some measurements of initial rigid avatar A1 in line with the real body;

- export the file of A1 in "obj" format.

Figure 4(a) compares the avatar A1 (in yellow) with the real scanned body (scanatar in green). Moreover, some details, such as genitalia position (specific height, bulging size) and the body standing inclination (forward and backward), require 3D Max assisted modification in getting the main parts with the same dimensions. In other words, avatar A1 should be adequate to conform to the scanatar.

\section{Rigid avatar modification}

During Step A2, the deformed avatar A2 was created on avatar A1 using 3ds Max after carrying out the following steps:

- import of A1, adding "push-up effects" according to a fixed value;

- change the height and state of the "genitalia" to the upward state;

- change the height of the buttock peaks by lifting soft tissue.

The simulated genitalia is considered as solid, nondeformed, and hard in software that does not allow one to simulate the functionality of virtual underwear, the performance of soft tissue, or the try-on test. The shape and position of the genitalia are very unstable in reality to calculate the height after lifting. To avoid this problem, we recommend considering the genitalia as a rounded sphere in the sagittal section, which includes the entire penis from peak to end, and the testicles. It can be a reference standard even if the genitalia position is changed. As shown in Figure 4(c), we can calculate the coordinates of the center location instead of calculating the peak of the penis (because the displacement distance of the testicles is ignored).

We chose avatar A1 as a middle type with $W_{G}=77.5 \mathrm{~cm}, \quad H_{G}=95.0 \mathrm{~cm}$, and $T_{G}=54.0 \mathrm{~cm}$. Firstly, the components of male genitalia and hip height were modified in $3 \mathrm{ds}$ Max using the "editable vertex" tool, as shown in Figure 4(b). Then, we built two spheres: the yellow sphere covering the genitalia of A1 without the push-up effect, and the green sphere for covering the genitalia of A2 after pushing up. Figure 4(c) illustrates the difference between the genitalia and buttocks of A1 and A2 from overlapping perspectives. The location of the two spheres represented the displacement of the genitalia. We simulated the state of the genitalia after largest lifting by $8.8 \mathrm{~cm}$ and the buttocks by $1.1 \mathrm{~cm}$ (according to our previous results, the range of the push-up effects of the genitalia is $2.1-8.8 \mathrm{~cm}$, and that of the back is $0.2-1.1 \mathrm{~cm}$ ).

So, the deformed avatar A2 with the predictable push-up effect (functional underwear) is designed. As we can see, the green sphere after lifting looks more prominent and the buttocks look firmer due to the pubic bone being closely connected to the whole genitalia. Therefore, the lifting effect not only has good support and wearing comfort but also produces a good visual effect - highlighting the male soft tissue.

Finally, in this section two types of avatars are proposed for the next virtual try-on test: initial rigid avatar A1 with the natural fall genitalia and buttock shape and deformed rigid avatar A2 with the genitalia lifted by $8.8 \mathrm{~cm}$ and the buttocks lifted by $1.1 \mathrm{~cm}$. All avatars (a)

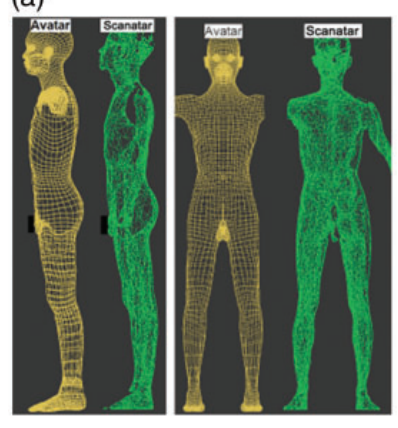

(b)

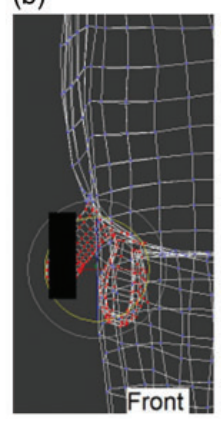

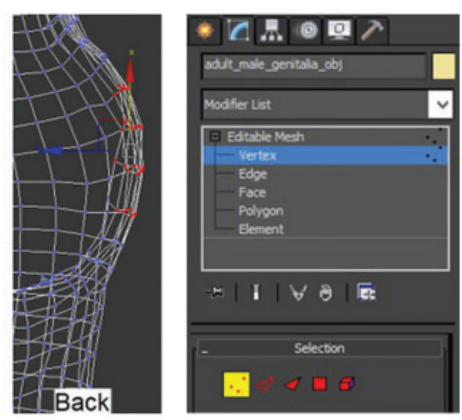

(c)

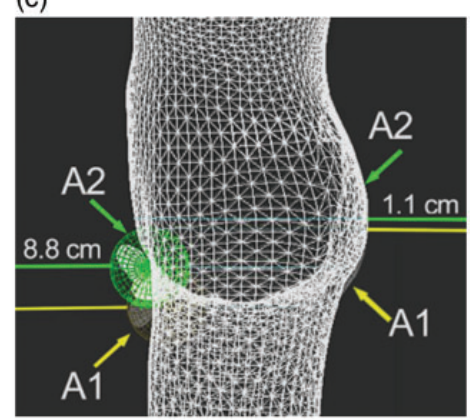

Figure 4. Comparison of established avatar AI and the scanatar (a); screenshots of modifying the genitalia and hip by $3 \mathrm{ds}$ Max (b); profile view of avatars with spheres covering the genitalia of avatar Al (yellow) and deformed rigid avatar A2 (green, after lifting) (c). (Color online only.) 
will be imported into the CLO software in "obj" format for subsequent simulation works.

\section{Simulation of knitted materials}

\section{Adjustment of calculated parameters}

In order to further verify the validity and reliability of the simulation, it is necessary to compare the testing data with the real experiment directly and to test the pressure in VR. Figures 5(a) and (b) show the two operation panels - "detail" and "simulation properties" - in which we entered the data to simulate the real knitted materials properties. The high-precision KES instrument is used to test the performance of the knitted material to put the KES parameters into the CLO system. At the same time, we reduced the length of the knitted material strips gradually around the body, which is similar to a negative ease $E$ in pattern blocks to obtain increasing pressure values in the real test and to make underwear tighter. This $E$ range is approximately $-20-0 \% . E_{\max }$ is the negative ease $E$, which can produce the maximum acceptable pressure on the body parts, for example, as shown in Figure 5(c).

CLO is equipped with "object browser" - "property editor" - fabric "physical property" - "detail" for the simulation, as we can see in Figure 5(a). The "detail" option has two sets of numbers (linked): the upper range is $0-100$ (without units) and the lower one is $0-10,000$ (without units of textile material parameters). We have chosen the lower one (stiffness), which is an accurate multi-digit number, and the following data can be kept only at four or five digits when ensuring that the pressure value is close to the real value. Then, we entered the measured real material shrinkage into "shrinkage-warp/weft, \%" in "simulation properties" plane, and density in the "thickness" plane for better displaying knitted material parameters.

During Step B1, in order to ensure the accuracy of knitting material performance simulation, the algorithm is determined for adjusting the KES parameters and pairing the pressure values of the real body and the virtual avatar, which are measured on the four body parts:

- enter the KES parameter $R T$, where the reference indicator is $100 \%$;

- enter the KES parameter $G$, where the reference indicator is 1 ;

- enter the thickness, density, and shrinkage directly in natural size into the options.

After the experiments, the equations are obtained, as shown in Table 4. Besides, according to recommendations, the equations from Table 4 should be used based on different compression performances $C P$ of the knitted material. Factor " $a$ " is added into the equations for further optimization.

The larger the values of indexes $R T$ and $E M T$, the better the recoverability and extendibility, the better the elasticity, and the weaker the pressure feeling exerted in general. So, we use the reciprocal values of $R T$ and $E M T(1 / R T$ and $1 / E M T$ in the equations) to decrease the values of material with good elasticity in order to decrease the results of $Y 1$, which is in line with the real effect. The average value of $F_{\max }$ has little influence on the multi-digit number of the knitted material simulation, but has an important reference value (the rigidity of material under real $E_{\max }$ ). After the preliminary test of the performance of the virtual materials, the general equations are calculated. $Y 1, Y 2$ are between 1983 and 9271; the larger the value, the stronger the pressure that will arise in the simulation.

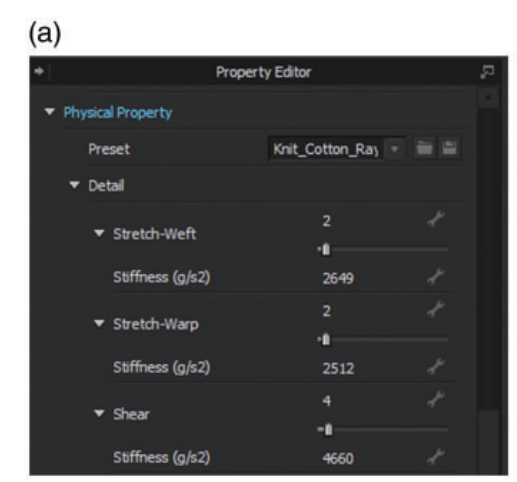

(b)

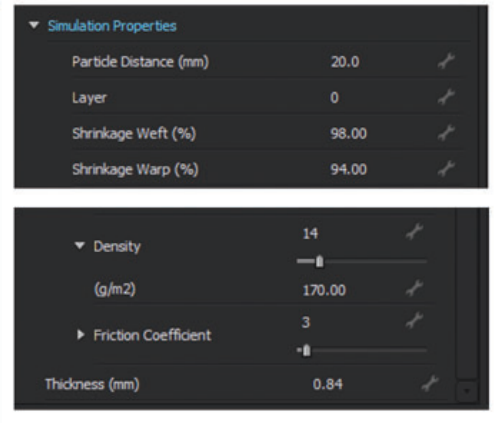

(c)

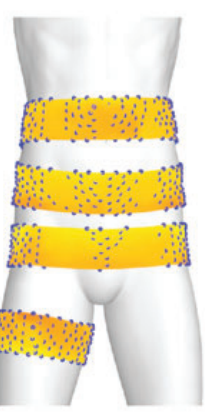

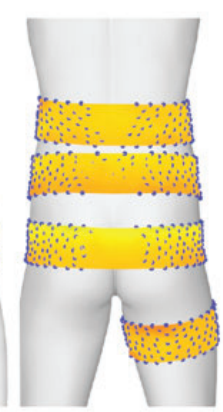

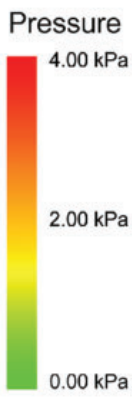

Figure 5. A panel of the virtual knitted material physical property (a); simulation properties panel (b); virtual pressure on avatar AI (c). 
Table 4. Algorithm for virtual knitted materials

\begin{tabular}{lll}
\hline KES parameters & CLO parameters & Equations \\
\hline$R T_{\text {warp }}, E M T_{\text {warp }}$ & Stretch-warp $Y \mathrm{I}$ & $Y \mathrm{I}=\left(\mathrm{I} / R T_{\text {warp }}+\mathrm{I} / E M T_{\text {warp }}\right) I 0^{5}+F_{\text {max.warp }}+a$ \\
$R T_{\text {weft }}, E M T_{\text {weft }}$ & Stretch-weft $Y 2$ & $Y 2=\left(I / R T_{\text {weft }}+I / E M T_{\text {weft }}\right) 10^{5}+F_{\text {max.weft }}+a$ \\
$G_{\text {warp }}, G_{\text {weft }}$ & Shear $Y 3$ & $Y 3=\left(G_{\text {warp }}+G_{\text {weft }}\right) 10^{4}$ \\
\hline
\end{tabular}

Notes: $R T$ is the recoverability, \%, EMT is the elongation under $500 \mathrm{cN} / \mathrm{cm}, \% ; F_{\max }$ is the same as $F($ Emax $)$, which is the load/tension force at $E_{\max }$, where the range is $6.1-448.5 \mathrm{gf} / \mathrm{cm}$; $G$ is the shear rigidity in the bias direction, where the higher the value the harder the shearing property; $Y \mathrm{I}$ is brought into the "stretch of stiffness-warp," without units; $Y 2$ is brought into the "stretch of stiffness-weft," without units; $Y 3$ is brought into the "shear of stiffness," without units; $a$ is the correcting factor, without units.

KES: Kawabata Evaluation System.

Table 5. Material data comparison (color online only)

\begin{tabular}{|c|c|c|c|c|c|c|c|}
\hline \multirow[b]{2}{*}{ Materials } & \multicolumn{2}{|c|}{ Real CP, $\mathrm{kPa} / \%$} & \multirow{2}{*}{$\frac{\text { Real pressure }}{\text { Avg. } P_{\text {B.max }}, \mathrm{kPa}}$} & \multirow{2}{*}{$\frac{\text { Virtual pressure }}{\text { Avg. } P_{V \text { max }}, \mathrm{kPa}}$} & \multirow{2}{*}{$\begin{array}{l}\text { Relative } \\
\text { error, \% }\end{array}$} & \multicolumn{2}{|c|}{ Calculated results } \\
\hline & Warp & Weft & & & & $Y I$ & $Y 2$ \\
\hline TI & 0.118 & 0.118 & 2.02 & 2.02 & 0.04 & 8421 & 8466 \\
\hline T2 & 0.166 & 0.149 & 2.33 & 2.13 & 8.68 & 8846 & 7893 \\
\hline T3 & 0.122 & 0.114 & 2.12 & 2.22 & 4.66 & 6131 & 4961 \\
\hline T4 & 0.110 & 0.099 & 1.81 & 2.06 & 13.57 & 8673 & 8247 \\
\hline T5 & 0.097 & 0.100 & 1.78 & 1.94 & 8.94 & 7431 & 9077 \\
\hline T6 & 0.080 & 0.083 & 1.60 & 1.68 & 5.45 & 7505 & 7109 \\
\hline T7 & 0.029 & 0.038 & 0.75 & 0.68 & 9.66 & 2070 & 1983 \\
\hline T8 & 0.026 & 0.030 & 0.61 & 0.65 & 6.29 & 2530 & 2437 \\
\hline T9 & 0.136 & 0.114 & 2.10 & 2.04 & 2.90 & 9271 & 5280 \\
\hline TIO & 0.034 & 0.054 & 0.95 & 1.06 & 11.93 & 3717 & 3451 \\
\hline TII & 0.097 & 0.082 & 1.89 & 1.73 & 8.37 & 4691 & 5021 \\
\hline TI2 & 0.101 & 0.078 & 1.93 & 1.75 & 9.58 & 4503 & 4432 \\
\hline TI3 & 0.090 & 0.074 & 1.69 & 1.54 & 8.40 & 4027 & 4219 \\
\hline TI4 & 0.087 & 0.077 & 1.79 & 1.72 & 3.86 & 3006 & 2991 \\
\hline Mean & 0.092 & 0.086 & 1.67 & 1.66 & 7.31 & 5773 & 5398 \\
\hline
\end{tabular}

However, the virtual pressure calculated by Equations (2) and (3) still has some errors compared with the real pressure because of the physical properties of materials. Therefore, value " $a$ " $(a \neq 0)$, which should be added to adjust the final result, needs further accurate formulation of the equations and will be discussed in the following sections.

\section{Simulation of knitted materials based on compression performance}

According to the equations above, constant values of " $a$ " are added to continue the experiment according to compression performance $(C P)$ to optimize the results. Table 5 shows the real $C P$ from the strongest to the weakest level with red-orange-green-blue colors and the maximum pressure $P_{B \text {.max }}$ under materials on the real body (B) and $P_{V \cdot \max }$ on the virtual avatar $(\mathrm{V})$ when the underwear is designed with the same $E_{\max }$.
Through multiple numerical modifications and pressure value tests, the hypothesis of the effect of the $C P$ of knitted materials on the pressure value is verified. The results of the $Y 1, Y 2$ calculation shown is in Table 5. As we can see, the $P_{V \cdot \max }$ values are smaller than the real data $P_{B \text {.max }}$, with the relative error $\delta=7.31 \pm 3.66 \%$. So, the maximum pressure on the human body under stretching material should be measured at first, then to find the $C P$, choose $a$ to correct Equations (2) and (3). The constant $a$ is dependent on $C P$ levels, and we can choose the integer $[0,1000,2000,3000$ without unit] for Equations (2) and (3)

The relative errors of the two kinds of materials (T4 and T10) are large. These two materials are mainly made of superfine fibers - long-staple cotton (80s) and micro modal (60s), single rib, where the thicknesses are 0.62 and $0.70 \mathrm{~mm}$, and they are thinner than other materials. In addition, the contact surface with the human body surface is smoother than that of 
others; the mean frictional coefficients MIU are 1.19 and 1.16 (the average of other materials is 0.24), which indicates a smoother surface; the surface roughness SMD values are 1.96 and $1.11 \mu \mathrm{m}$ (the average of other materials is 3.34), indicating a geometrically rougher surface. ${ }^{52}$ Based on a variety of different factors, the measurement of pressure values will be affected to a certain extent.

Based on the results above, it is found that the virtual average pressure under the virtual material is very similar to the real one, with the range of relative error being $0.04-14.00 \%$. Due to the influence of the soft tissue parts and the bones, as well as the physical properties of the material, the results of the tested materials have been used to minimize the experimental errors. Next, we will demonstrate the feasibility of this algorithm through a variety of simulations.

\section{Simulation of the performance of knitted materials}

During Step B2, we tested the average virtual pressure arising under different materials under its elongations $E=5 \%, 10 \%, 15 \%, 20 \%$ in the warp and weft; the virtual pressure values (nearly 5000 times) are measured on four parts of avatar A1. Then, further tests of pressure measurement were conducted on different parts of the avatar and material deformation, as shown in Figures 6(b) and (c).

The scatter plot of real and virtual pressure comparison is as shown in Figure 6(a), where most data are concentrated in the lower left corner of the graph and approximately less than $1.00 \mathrm{kPa}$. In total, the average differences (absolute error $\Delta$ ) between virtual and real pressure are $\Delta=0.12,0.20,-0.01,0.20 \mathrm{kPa}$ under elongations $E=5 \%, 10 \%, 15 \%, 20 \%$, respectively. As can be seen from the results, the virtual pressure also belongs in the reasonable comfort pressure range as in the real tests, and both values also have a linear relationship. Figure 6(b) shows that the differences between pressure $P_{V \cdot \max }$ on the natural waist, waistband, hip, and thigh are smaller. The virtual pressure is stronger than the real one on the real buttocks, and the real pressure is stronger on the waistband (at the anterior superior iliac spine). The relative error calculated on the four parts is $\delta=8.27 \pm 6.76 \%$; the absolute errors are $0.23 \mathrm{kPa}$ on the hip, $0.10 \mathrm{kPa}$ on the waistband, $0.08 \mathrm{kPa}$ on the thigh, and $0.07 \mathrm{kPa}$ on the natural waist. Figure 6(c) shows comparisons of the deformations of virtual materials in CLO and the real $E_{\max }$ designed: all virtual results are linearly correlated with the real value of $E_{\max }$, but a little larger.

Furthermore, the Shapiro-Wilk test of SPSS was applied for the evaluation of the normality of distribution. We investigated the pressure in reality $P_{B}$ and in simulation $P_{V}$ produced by stretching the materials by $5 \%, 10 \%, 15 \%, 20 \%$, and the maximum of $20.5 \%$. We used the following symbols to mark the samples: for example, $P_{B .5}$ represents the real pressure measured under the elongation of material at $5 \%$ and $P_{V .5}$ represents the virtual pressure under the same elongation. The significance of five sets of data are between 0.059 and 1.000 , so the data has normal distribution.

Then, five sets of data for pressure were examined separately by paired samples $t$-tests, as shown in Table 6.

Table 6 shows that the differential value of all pairs $1-5$ is $-0.011-0.175 \mathrm{kPa}$. According to the statistical principle, $\alpha=0.001$ is used as a reference when sig. is $0.011-0.959$. We can find that the $P_{B . E}$ and $P_{V . E}$ with different $E$ values have strong linear correlation. Moreover, there are strong correlations between the pressure (red and orange colors points) values of $P_{B . E}$ and $P_{V . E}$ at large material elongation states, so, the virtual pressure value is closer to the real one in the case of large elongations of material.

Through the calculation and simulation in Step B2, the digital knitted materials should be based on the

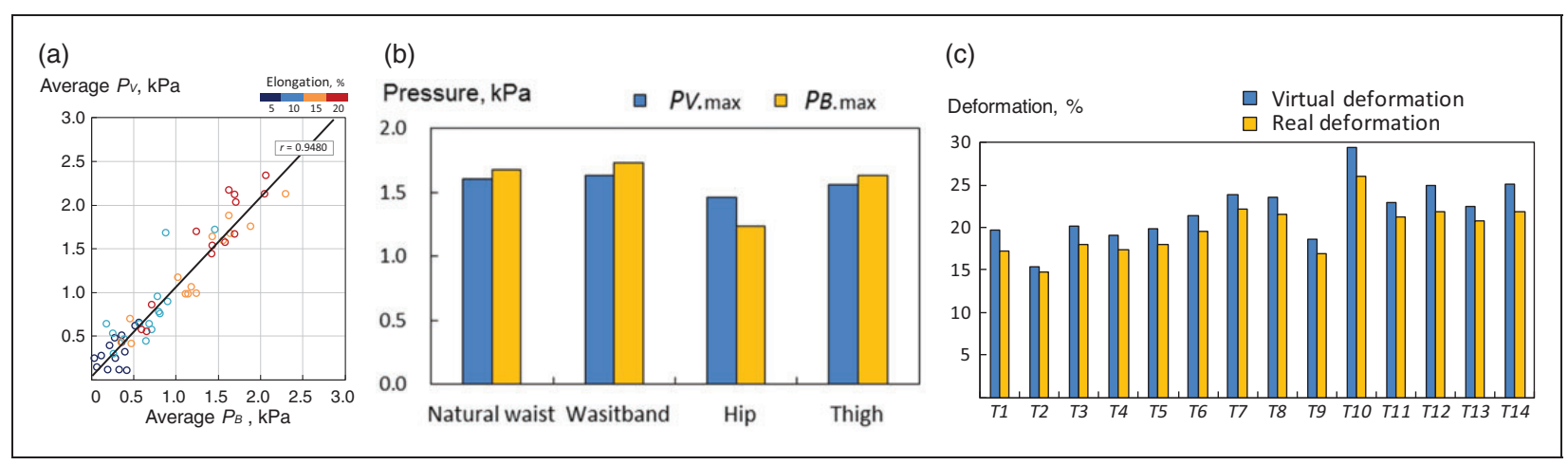

Figure 6. Virtual pressure under knitted material elongation (a); comparison of the virtual and real pressure on different parts (b); comparison of real and virtual deformations (c). 
Table 6. Test of paired samples

\begin{tabular}{|c|c|c|c|c|c|c|c|c|c|}
\hline \multicolumn{7}{|c|}{ Differences between paired samples $\left(P_{B . E}-P_{V . E}\right)$} & \multicolumn{3}{|c|}{ Correlations between paired samples $\left(P_{B . E}\right.$ and $\left.P_{V . E}\right)$} \\
\hline & & Mean & Std. dev. & $t$ & $d f$ & $\begin{array}{l}\text { Sig. } \\
\text { (2-tailed) }\end{array}$ & & $\begin{array}{l}\text { Correlation } \\
\text { coefficient }\end{array}$ & Sig. \\
\hline Pair I & $P_{V .5}-P_{B .5}$ & 0.041 & 0.161 & 0.956 & 13 & 0.357 & $P_{B .5}$ and $P_{V .5}$ & 0.571 & 0.033 \\
\hline Pair 2 & $P_{V .10}-P_{B .10}$ & 0.125 & 0.264 & 1.767 & 13 & 0.101 & $P_{B .10}$ and $P_{V .10}$ & 0.784 & 0.001 \\
\hline Pair 3 & $P_{V .15}-P_{B .15}$ & 0.002 & 0.168 & 0.053 & 13 & 0.959 & $P_{B .15}$ and $P_{V .15}$ & 0.954 & 0.000 \\
\hline Pair 4 & $P_{V .20}-P_{B .20}$ & 0.175 & 0.210 & 3.005 & 12 & 0.011 & $P_{B .20}$ and $P_{V .20}$ & 0.945 & 0.000 \\
\hline Pair 5 & $P_{V \cdot \max }-P_{B \cdot \max }$ & -0.011 & 0.139 & -0.287 & 13 & 0.779 & $P_{B \cdot \max }$ and $P_{V \cdot \max }$ & 0.965 & 0.000 \\
\hline
\end{tabular}

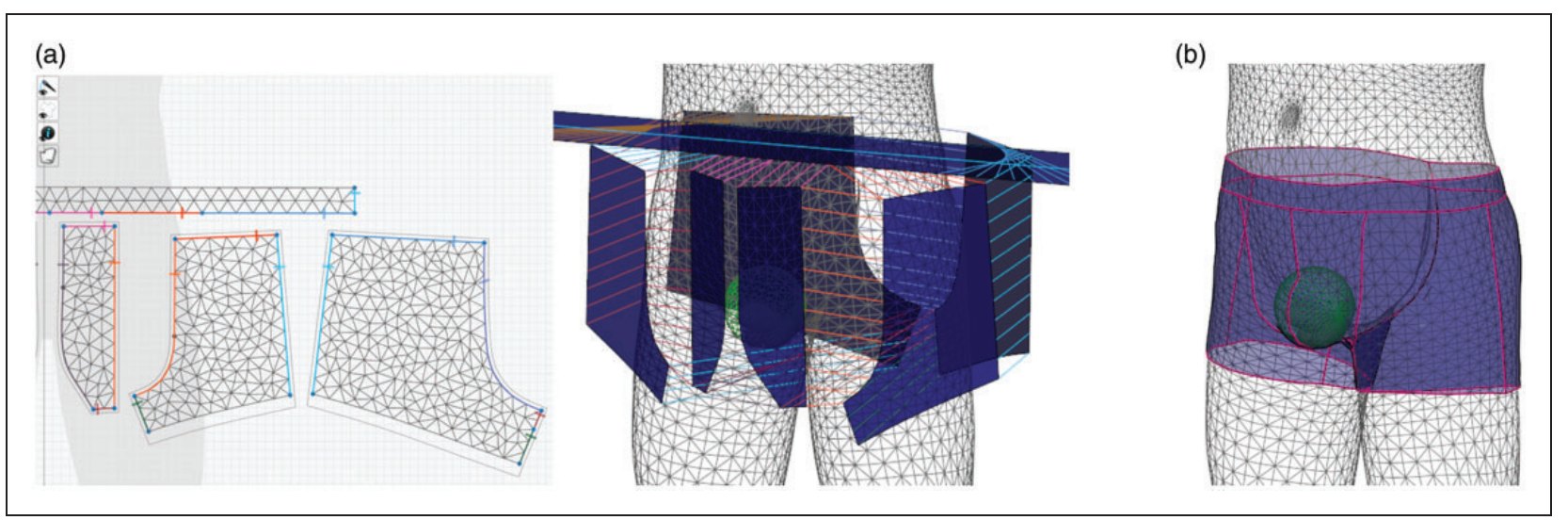

Figure 7. Underwear pattern block before virtual sewing (a); proper virtual try-on (b).

following parameters: KES properties $(R T, E M T, G$, and $F$ ); the compression performance $C P$; the thickness; and the density. It can be seen that the results of the virtual materials are extremely similar to the real prototypes and they produce adequate pressure without noticeable differences. Therefore, the algorithm we proposed can be applied to the next simulation experiment in Step B3.

\section{Evaluation of system "avatar-underwear"}

\section{Constructing virtual underwear}

During Step C1, 2D the basic pattern blocks were imported into CLO, as shown in Figure 7. After defining half of the pattern, the other half is copied by the "instance pattern" function. Then, we arranged each pattern block to the corresponding location around the avatar and joined them together using the virtual "sewing tools"(Figure 7(a)).

To achieve the tightness effect, the dimensions of compression underwear should be smaller than the body measurements. However, an inelastic clothing size smaller than the avatar usually penetrates the avatar during try-on (Figure 7(b))..$^{14,53}$ Therefore, it is necessary to input the numerical values of the elastic knitted fabric to ensure that the virtual material is elastic at first. Secondly, during virtual try-on the coordinates of the avatar and the underwear must strictly correspond one-to-one, so that the virtual underwear can be properly wrapped on the corresponding part of the avatar, not breaking into the interior or falling off. For example, the position of the waistband and bottom should be fixed on the avatar surface with the tool "tack on avatar."

\section{Virtual try-on}

During Steps B2 and C2, we carried out the preliminary structural test of the basic underwear type based on the pressure and deformation observations:

- design the underwear for virtual sewing;

- adjust the avatars with medium size $\mathbf{M}$ for the try-on;

- import the parameters of knitted materials into the virtual system;

- carry out the preliminary observation and the objective evaluation according to the physical performance of virtual material in the CLO try-on. 
We build the basic underwear with the ease $E_{\max }$ for avatar A1. The mode of material "pressure" and "deformation" is used to observe the underwear models by CLO. Figure 8(a), "original" virtual underwear, illustrates that the crotch part is under excessive pressure and significant deformation in red or orange (in the bottom view). This situation means that the length of the hip or the crotch part is too short and leads to large deformation of the materials and pressure. So, the hip line should be optimized to avoid this problem, and the virtual deformation and pressure values in the crotch part become lower than before; the "optimized" version is shown on the right-hand side of Figure 8(a).

Through the optimization of the structural details, we compare and measure the changes of deformation and pressure values for the crotch part. The average pressure value decreased by $1.3 \mathrm{kPa}$ and the average deformation decreased by $17.1 \%$. We can see that the values after optimizing the crotch part are lower than before, as shown in Figure 8(b). Through the preliminary objective test of the underwear optimization in Step $\mathrm{C} 3$, the overall performance is good, which proves the feasibility of the simulation process.

\section{Digital replica of basic underwear}

After Steps A2, B2, and C2, in order to further evaluate and prove this simulation method, the variety of avatars used to conduct try-on tests are compared with the real data in Step D1.

To check the accuracy of underwear simulation with different materials and the matching degree of the avatars and real human bodies (scanatars), we selected four materials (T2, T4, T6, and T10) with different levels of compression performance $C P$ and put on the underwear of these materials on the avatars or the scanatars in $\mathrm{S}, \mathrm{M}$, and $\mathrm{L}$ sizes, respectively. The $\mathrm{S}, \mathrm{M}$, and $\mathrm{L}$ scanatars had the following measurements: $W_{G}=68.6 / 75.7 / 84.4 \mathrm{~cm}, \quad H_{G}=85.3 / 93.5 / 97.6 \mathrm{~cm}$, $T_{G}=46.7 / 50.6 / 56.5 \mathrm{~cm}$. Then, we measured the pressure at more than 60 points located on six parts of underwear, as shown in Figure 9(a). The virtual underwear has a triangular-mesh structure with many points (blue) that are available for pressure measurement. Due to the elongation of the knitted materials and the unevenness of the human body surface, some parts of the underwear cannot completely close to the avatar surface (red points on Figure 9(a)), such as the sagittal line between the buttocks, side, and the front groin. The average pressure is $1.14 \mathrm{kPa}$, and subjective criterion of comfort is above level-3, so the virtual underwear produces median pressure and provides a good feeling of comfort and is a little tight.

Figures 9(b) and (c) show the underwear made of different materials in $\mathrm{S}, \mathrm{M}$, and $\mathrm{L}$ sizes worn on the avatars (underwear is designed using the mass production approach (b), and for wearing on the testee (scanatars) (underwear is designed in the customized approach (c)).

Based on this try-on, the evaluation ratings are levels-2-5. The results showed that each avatar has appropriate size underwear, and the same material produces similar pressure distribution on each avatar. T10 with weak $C P$ produced the weakest pressure of $0.76 \mathrm{kPa}$, but it is higher than that of level-2 and is also tight. T2, T4, and T6 have scores that are higher than level-3, so the pressure and compression are relatively good for tight underwear. ${ }^{54}$ However, the pressure on the "genitalia" bottom and the waistband sides is strong. In the try-on simulation, each material has similar pressure values in the $\mathrm{S}, \mathrm{M}$, and $\mathrm{L}$ sizes.

Based on the simulation test, we created avatars (replicas) and virtual underwear, and our approach (a)

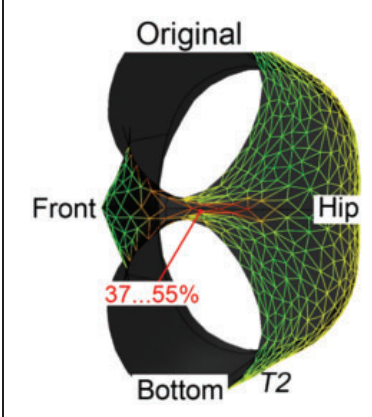

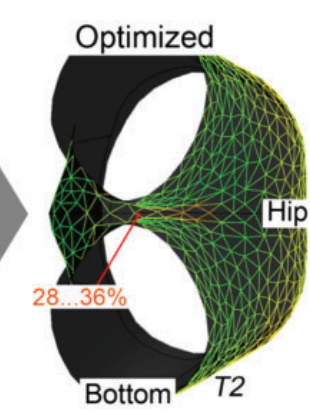

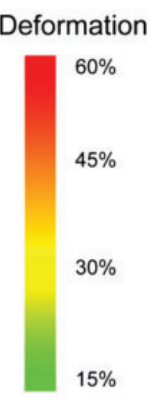

(b)

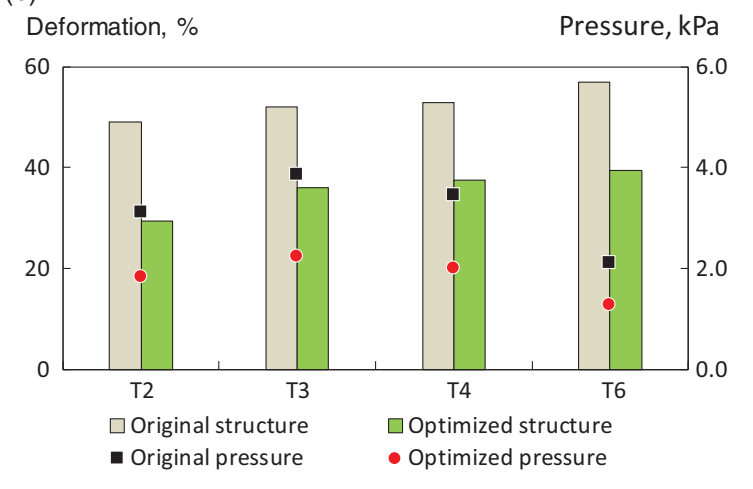

Figure 8. The underwear crotch part before and after optimized in the upward view (a); comparison of the original and optimized data only on the crotch part (b). (Color online only.) 


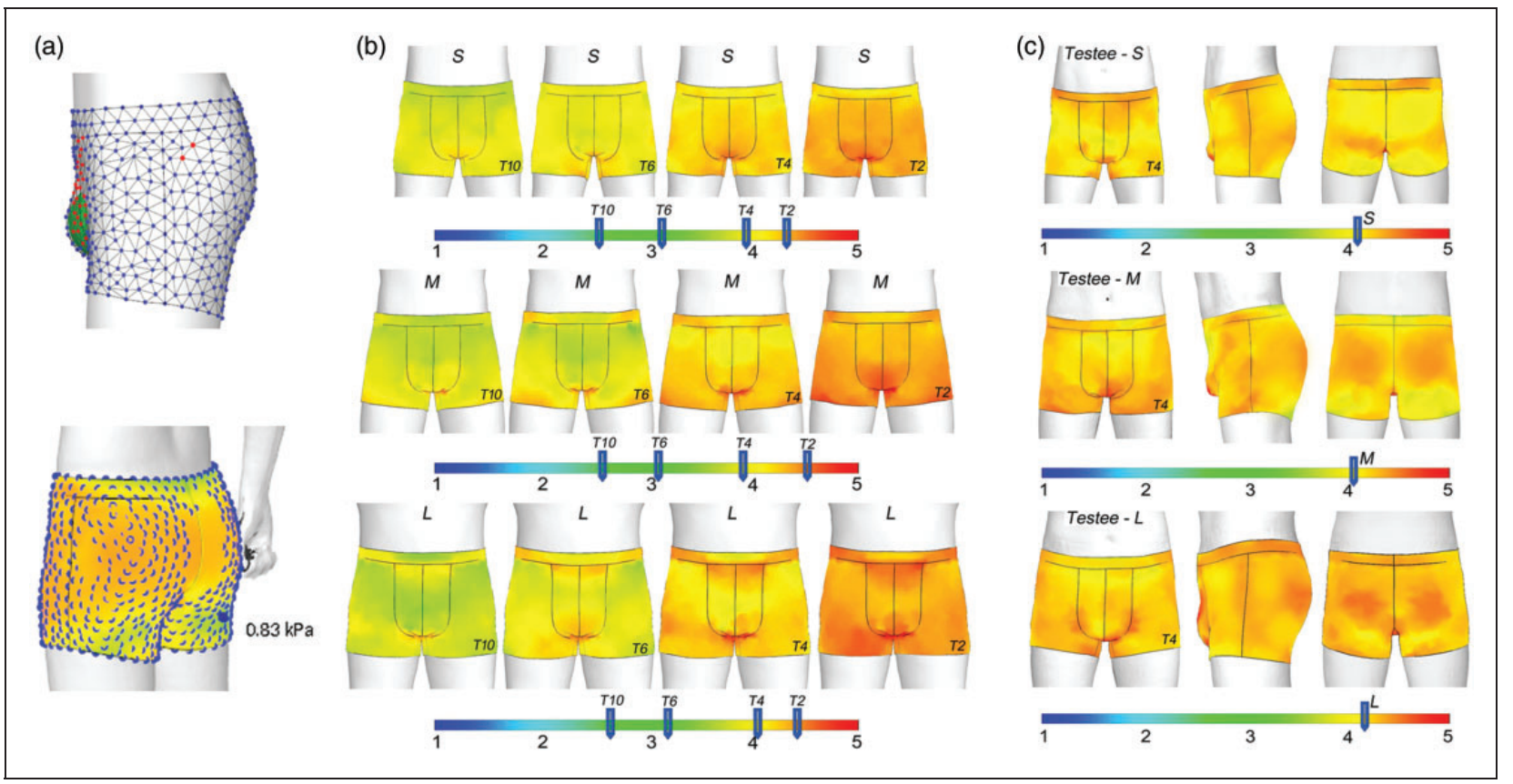

Figure 9. Measurement points (a); evaluation of underwear pressure distribution on the avatars (b); evaluation of underwear pressure distribution on the scanatars (c). (Color online only.)

can be used to design virtual underwear in different sizes and to complete the design directly through $3 \mathrm{D}$ software and predict comfort results; the average size of avatars/male bodies and individuals are well matched.

\section{Digital replica of functional underwear}

The virtual try-on of two types of underwear - basic and functional - is conducted. Both types of underwear are made of materials $\mathrm{T} 2, \mathrm{~T} 4$, and $\mathrm{T} 6$ with the $C P$ (at the strongest, strong, and weak levels, respectively) to see the push-up effects.

Figure 10 shows the wearing performance of the virtual underwear on the rigid avatars $\mathrm{A} 1$ and $\mathrm{A} 2$, and the real male testee of $M$ size. In our database, the $M$ size has the highest proportion rates of $64-73 \%$ according to the classification in Table $2 .{ }^{18} \mathrm{We}$ chose the real male testee (we have named him Testee-M) with average sizes of $W_{G}=76.8 \mathrm{~cm}$ and $H_{G}=92.9 \mathrm{~cm}$. The testee's profile photos with underwear is shown in Figure 10(c).

The background grid is $1 \mathrm{~cm} * 1 \mathrm{~cm}$, and we marked the lifting effect of the front genitalia as F1, F2, F3 and the lifting effects of the buttocks height as B1, B2, B3. All genitalia are placed in an upward state to compare.

As we can see from Figure 10(a), the bottom of the genitalia, the side of the waistband, and the buttocks are under strong pressure (the observable pressure in orange and red colors). Material T2 produced a lifting effect on the genitalia of $\mathrm{A} 1$, but for avatar $\mathrm{A} 2$ the pressure becomes weaker. Material T6 produced weaker pressure on the genitalia bottom, and other parts, because its $C P$ value weaker was weaker than that of $\mathrm{T} 2$.

Due to the lifting effect, both materials T2 and T6 produced strong pressure on the genitalia, and after lifting, the underwear provided good support. As Figure 10(c) shows, a contrast exists between the push-up underwear and the basic type.

Table 7 shows the results after comparison of the underwear.

As shown in Table 7, the underwear made of material T2 produced stronger pressure than that made of material T6, and provided an obvious lifting effect on the front and buttock. Moreover, from the feedback of the testee's wearing experience, the push-up type underwear has good support on the front and back, the underwear made of $\mathrm{T} 2$ is tighter than that made of T6, and the compression effect on the buttocks is also stronger than that of T6. The basic underwear T4 also has a good fit and compression feeling, but without lifting effects.

During Step D2, the evaluation of real experiments was conducted with three basic pairs of underwear (with $\mathrm{S}, \mathrm{M}, \mathrm{L}$ sizes) made of material $\mathrm{T} 4$, and four functional styles (with $\mathrm{S}, \mathrm{M}$, L sizes) made of $\mathrm{T} 2, \mathrm{~T} 4$, T6, T10 for three real testees: Testee- $\mathrm{S}\left(W_{G}=72.0 \mathrm{~cm}\right.$, $\left.H_{G}=88.7 \mathrm{~cm}\right) ; \quad$ Testee-M $\left(W_{G}=76.8 \mathrm{~cm}, \quad H_{G}=\right.$ $92.9 \mathrm{~cm})$; Testee-L $\left(W_{G}=90.4 \mathrm{~cm}, \quad H_{G}=110.9 \mathrm{~cm}\right)$. The real pressure has been measured on the hip (buttocks, middle of the gluteus maximus), around the 
(a)

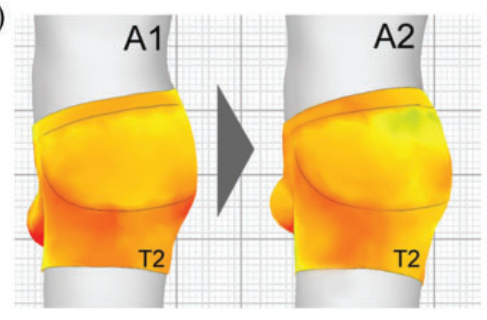

(b)

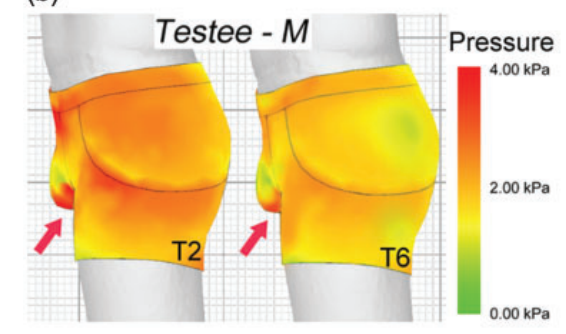

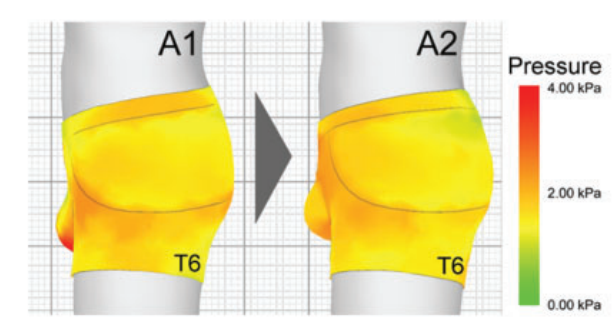

(c)

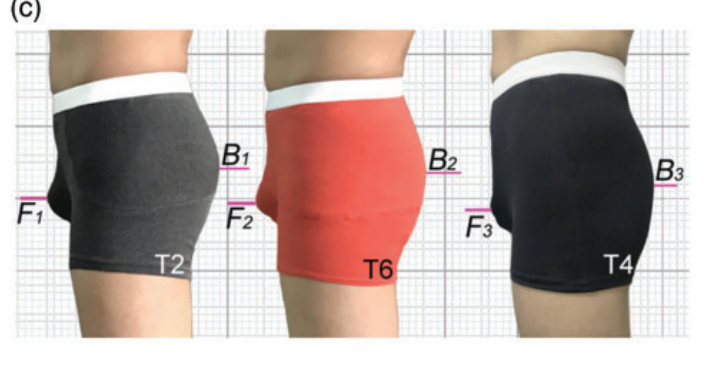

Figure 10. Push-up type underwear made of T2 and T6 worn on avatars AI and A2 (a); push-up type underwear made of T2 and T6 worn on scanatar M (b); underwear worn by Testee-M: push-up type underwear made of T2 and T6, basic underwear made of T4 (c). (Colors online only.)

Table 7. Comparison of the push-up effect

\begin{tabular}{|c|c|c|c|c|c|}
\hline & & \multicolumn{2}{|c|}{ Front (genitalia) } & \multicolumn{2}{|c|}{ Hip (buttocks) } \\
\hline & & $\mathrm{T} 2$ & T6 & $\mathrm{T} 2$ & T6 \\
\hline \multirow[t]{3}{*}{ Pressure, $\mathrm{kPa}$} & $\mathrm{Al}$ & 3.67 & 3.21 & 2.46 & 1.94 \\
\hline & $\mathrm{A} 2$ & 2.50 & 2.04 & 2.15 & 1.36 \\
\hline & Scanatar M & 3.75 & 2.71 & 2.07 & 1.58 \\
\hline Height, cm & Scanatar M & $\mathrm{FI}=77.5$ & $F 2=76.9$ & $\mathrm{BI}=8 \mathrm{I} .8$ & $B 2=81.4$ \\
\hline $\begin{array}{l}\text { Offset from } \\
\text { T4, cm }\end{array}$ & Scanatar M & +2.3 & +1.7 & +1.7 & +1.3 \\
\hline
\end{tabular}

waistband, and the bottom in a static standing posture and four dynamic postures (Figure 11).

The average results of the measurements are shown in Figure 12.

As Figure 12(a) shows, under the same movements in two tests (virtual and real), the simulation effect is the best when standing naturally. The difference between the pressure measured in the virtual system (on the avatar, the scanatar) and with the real testee is not significant, and the absolute error is $0.09 \mathrm{kPa}$ (the relative error is $4.64 \%$ ).

In Figure 12(b), compared with the pressure measured when standing naturally, it is slightly unstable in dynamic postures. The absolute errors on the waistband, hip, and thigh parts are $0.18,0.15$, and $0.09 \mathrm{kPa}$ (the relative errors are $7.33 \%, 6.99 \%$, and $6.01 \%$ ), respectively. However, the tests did not show completely different results or significant errors because all measurements are within the acceptable comfort range. So, the underwear designed with the help of the virtual systems has extremely similar pressure to that of the real testee.

Furthermore, as Figure 13 shows, the subjective evaluation by using the scale from 1 "very uncomfortable" to 5 "very comfortable" is used to get feedback of the waistband, buttocks, crotch, side, and thigh parts. ${ }^{55}$ The average rating of each part is 4.2 and the average cumulative rating is 25.1 (the top rating is 30 ). There are good average ratings at the front pouch (4.1) and hips (4.7). So, the underwear samples designed by the virtual method have a good wearing experience in the subjective test. It can also be proved that the measured comfort pressure is within the comfort range.

By comparing the results obtaining with the avatars in VR and the real several male testees, it is obvious that the results of the virtual try-on application with multiple human body shapes and various knitted materials are completely adequate for the real wearing effect, as are the results of pressure and material deformation in static and dynamic conditions.

In VR we can change and accurately adjust and join many parameters related to $2 \mathrm{D}$ patterns and the $3 \mathrm{D}$ performance of avatars and underwear. Moreover, the simulation of underwear in the same size but from different knitted materials can be completed quickly only by modifying the numerical values of the knitted materials properties, without repeating the material cutting and making samples. In the dynamic, the virtual system has unified stable environmental parameters, and errors 


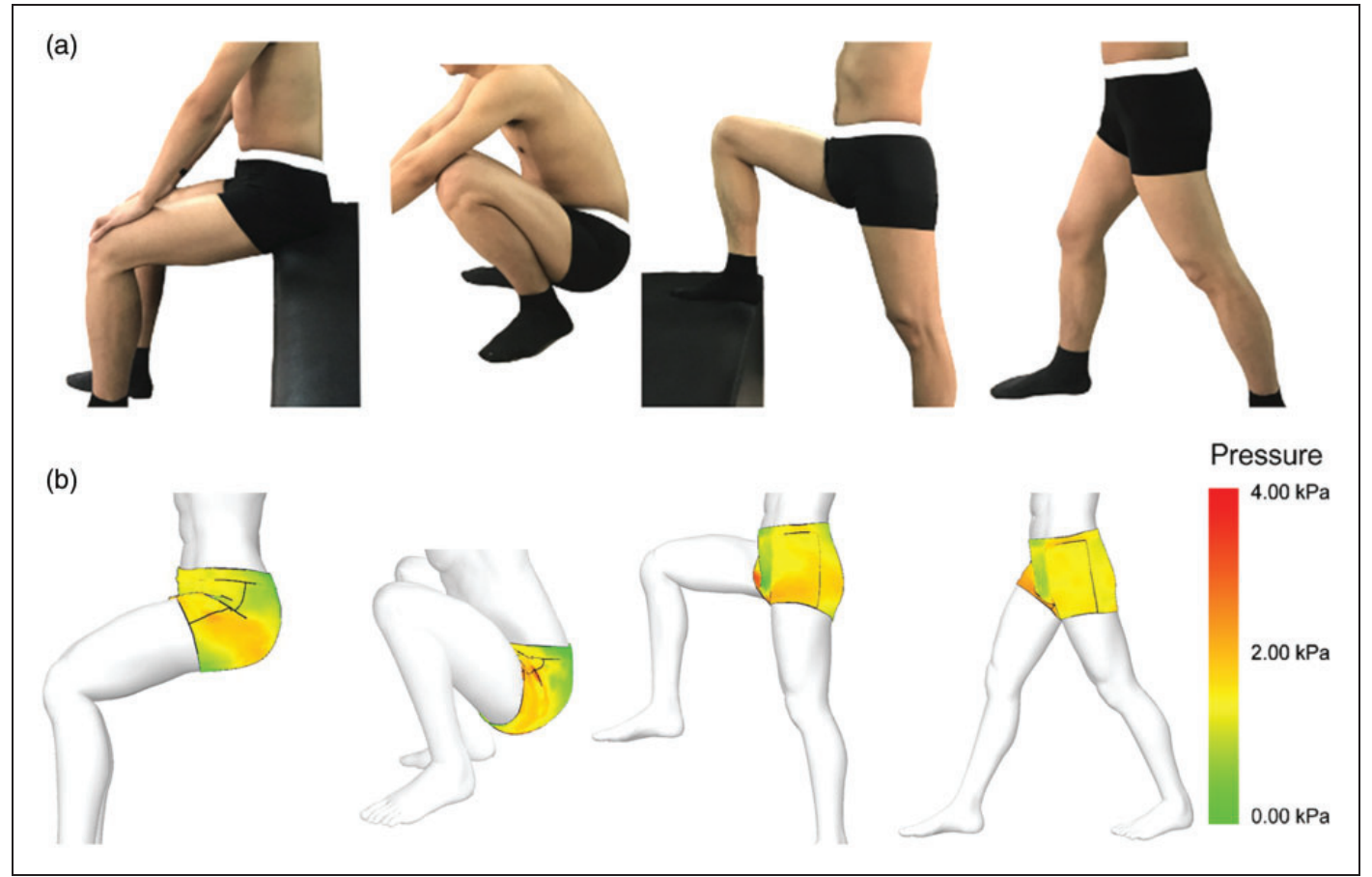

Figure II. The try-on of four postures.

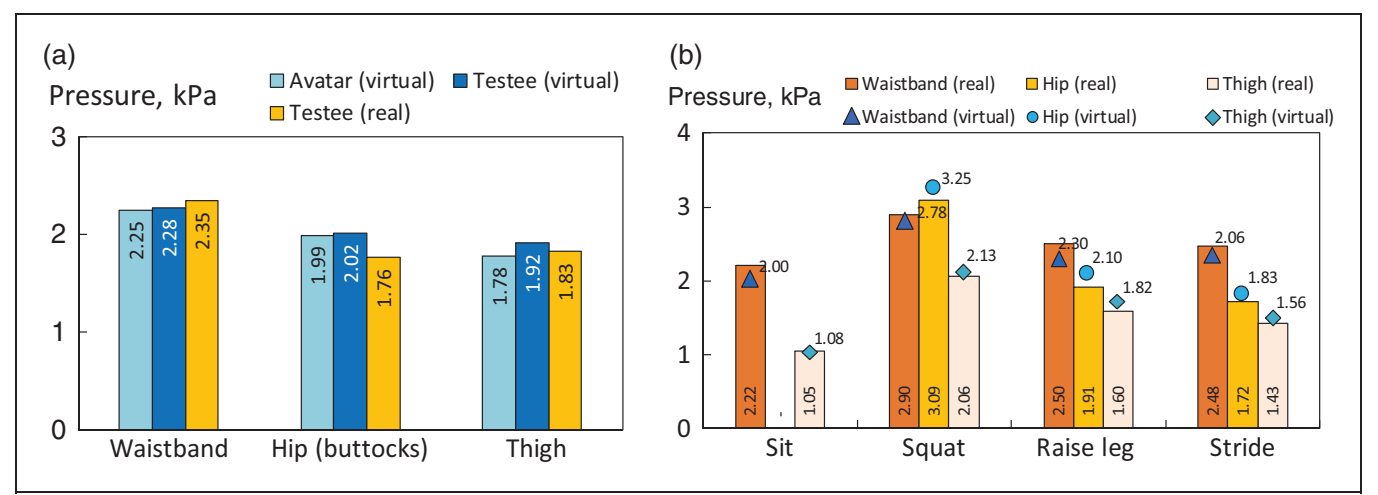

Figure 12. Comparison of virtual and real pressure of basic underwear T4 in a static standing position (a); comparison of average pressure of basic underwear T4 and underwear T2, T4, T6, and TI0 for four postures (b).

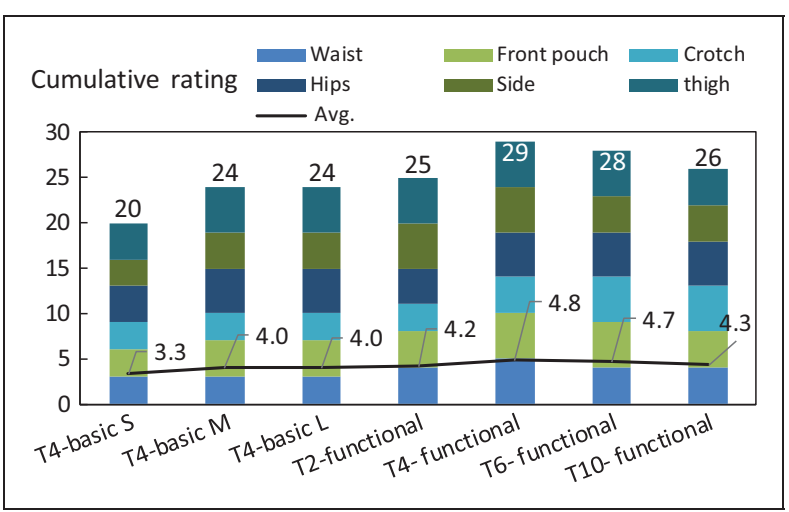

Figure 13. Rating of subjective evaluation. will not be caused due to different testees' non-standard dynamic posture or under the influence of environmental and human factors.

\section{Conclusion and discussion}

In this study, we have proposed a method of digital replica generation that is related to elastic knitted materials and an avatar of the male body. We build up and prove the new algorithm of comfort evaluation depending on the virtual material properties. Through the evaluation we found out that only some small errors exist between the virtual and the real results. The errors of the pressure prediction can be attributed to the 
intrinsic difference between the avatar surface and the soft tissue of the real human.

The proposed method of virtual design allows quick and accurate simulation of the real situation when the parameters of the virtual system are adjusted. The stability and the efficiency of the virtual environment are obvious.

All simulations are verified through virtual experiments. The digital replicas of underwear and knitted materials match the avatars well; this proves that the simulation of the push-up effect that is produced by stretching the material of underwear is practicable to meet the pressure data in the real test; the simulation process for achieving good virtual underwear has been verified. We recommend using the pressure rating method with a scale to provide a simple understanding of the pressure comfort influence on the design results. Moreover, we can infer what compression the knitted material will produce on the human body, and design the appropriate pressure according to the acceptance degree of human perception. However, at present, in the actual application, the precise push-up/lift value of the soft tissue cannot be accurately predicted. Only the developed virtual try-on evaluation can reach a conclusion without limitation. However, it is certain that underwear with the same functional structure but different materials will produce different push-up effects.

The limitations are as follows: (a) the soft tissues of the human body need more complex simulation methods; (b) textile materials parameters are only used to simulate the negative ease in underwear pattern blocks.

For future work, virtual simulation is a critical technology that can improve the efficiency of design and realize the virtual fitting system by using digital replicas of the male body, the textile materials, and the apparel. It can be used by designers and consumers to order, to buy, and to evaluate the comfort of online compressed underwear in advance. To improve the education database, it is necessary to scan more human bodies and to optimize the sizes of avatars to simulate more knitted materials to increase the content of the digital library, and further verify the algorithm of underwear design with stronger compression. Future works will involve other styles of compression apparel, and this method will be tested in a variety of styles.

\section{Declaration of conflicting interests}

The authors declared no potential conflicts of interest with respect to the research, authorship, and/or publication of this article.

\section{Funding}

The authors disclosed receipt of the following financial support for the research, authorship, and/or publication of this article: This work has been fully supported by the Ministry of Science and Education of Russian Federation under project number 2.2425.2017/4.6 "Development of software for virtual design of system "body - clothes" in static and dynamic and for virtual try-on FashionNet.

\section{ORCID iDs}

Zhe Cheng (D) https://orcid.org/0000-0002-2386-1060

Victor Kuzmichev (D) https://orcid.org/0000-0001-5625-0162

\section{References}

1. Wu X and Kuzmichev VE. Study on the body girth dynamic size for wetsuit ease design. IOP Conf Ser Mater Sci Eng 2018; 459: 012085.

2. Pons MG, Pujades S, et al. Clothcap: seamless 4d clothing capture and retargeting. ACM Trans Graph 2017; 36: 73.

3. Lin YL and Wang MJ. The development of a clothing fit evaluation system under virtual environment. Multimedia Tool Appl 2016; 75: 7575-7587.

4. $\mathrm{Hu} \mathrm{ZH}$, Li X, et al. Examining collaborative filtering algorithms for clothing recommendation in e-commerce. Text Res J 2018; 89: 2821-2835.

5. Hong Y, Bruniaux $\mathrm{P}$, et al. Design and evaluation of personalized garment block design method for atypical morphology using the knowledge-supported virtual simulation method. Text Res $J$ 2018; 88: 1721-1734.

6. Lee YA. Anthropometry, apparel sizing and design 11computer design and digital fit of clothing. UK: Woodhead Publishing, 2014, pp.305-3198.

7. Sook Cho Y, Tsuchiya K, et al. Computerized pattern making focus on fitting to $3 \mathrm{D}$ human body shapes. Int J Clothing Sci Technol 2010; 22: 16-24.

8. Abtew MA, Bruniaux P, et al. Development of comfortable and well-fitted bra pattern for customized female soft body armor through 3D design process of adaptive bust on virtual mannequin. Comput Ind 2018; 100: 7-20.

9. Lu S, Mok PY, et al. A new design concept: 3D to 2D textile pattern design for garments. Comput Aid Des 2017; 89: $35-49$.

10. Chen X, Tao X, et al. Control and optimization of human perception on virtual garment products by learning from experimental data. Knowl Base Syst 2015; 87: 92-101.

11. Mert E, Psikuta A, et al. A validation methodology and application of 3D garment simulation software to determine the distribution of air layers in garments during walking. Measurement 2018; 117: 153-164.

12. Colaianni M, Siegl C, et al. Anisotropic deformation for local shape control. Computat Vis Media 2017; 4: 305-313.

13. Popescu G, Olaru S, et al. Innovative design and simulation of clothing products for children with atypical changes in conformation and posture. DE Redactie 2017; 1: 63-68.

14. Duan L, Yueqi Z, et al. Automatic three-dimensionalscanned garment fitting based on virtual tailoring and geometric sewing. J Eng Fiber Fabric 2019; 14: 1558925018825319 . 
15. $\mathrm{Hu} \mathrm{P}, \mathrm{Ho}$ ESL, et al. A new method to evaluate the dynamic air gap thickness and garment sliding of virtual clothes during walking. Text Res $J$ 2018; 89: 4148-4161.

16. Cheng $\mathrm{Z}$ and Kuzmichev VE. Classification of male lower torso for underwear design. IOP Conf Ser Mater Sci Eng 2017; 254: 172007.

17. Wu YY, Mok PY, et al. An investigation on the validity of $3 \mathrm{D}$ clothing simulation for garment fit evaluation In: proceedings of the IMProVe 2011 international conference on innovative methods in product design, (ed. Gianmaria Concheri), Venice, Italy, pp. 15-17 June 2011, pp. 463468. Venice: UniversitÁ degli Studi di Padova.

18. Cheng $\mathrm{Z}$ and Kuzmichev VE. Research on the male lower torso for improving underwear design. Text Res $J$ 2018; 89: 1623-1641.

19. Tao X, Chen X, et al. A customized garment collaborative design process by using virtual reality and sensory evaluation on garment fit. Comput Ind Eng 2018; 115: 683-695.

20. Liu K, Zeng X, et al. Fit evaluation of virtual garment try-on by learning from digital pressure data. Knowl Base Syst 2017; 133: 174-182.

21. Thomassey S and Bruniaux P. A template of ease allowance for garments based on a 3D reverse methodology. Int J Ind Ergon 2013; 43: 406-416.

22. Jiang Y, Guo R, et al. Cloth simulation for Chinese traditional costumes. Multimedia Tool Appl 2019; 78: 1-26.

23. Cheng Z, Kuzmichev VE, et al. Development of knitted materials selection for compression underwear. AUTEX Res J 2017; 17: 177-187.

24. Cheng Z and Kuzmichev VE. Digital twin and men's underwear design. IOP Conf Ser Mater Sci Eng 2018; 459: 012075.

25. Lin SH, Johnson RR, et al. Fitting simulation evaluation on personalized avatars. J Text Eng Fashion Technol 2018; 4: 123-128.

26. Kim DE. Psychophysical testing of garment size variation using three-dimensional virtual try-on technology. Text Res $J$ 2016; 86: 365-379.

27. Kuzmichev VE, Tislenko IV, et al. Virtual design of knitted compression garments based on bodyscanning technology and the three-dimensional to two-dimensional approach. Text Res $J$ 2018; 89: 2456-2475.

28. Popescu G, Olaru S, et al. Innovative technologies for the design and simulation of children's clothing products using anthropometric data obtained by 3D standardized scanning. Industria Textila 2017; 68: 95.

29. Yu A and Yick KL. Engineering of high-performance textiles-12 compression and stretch fit garments. UK: Woodhead Publishing, 2018, pp.279-303.

30. Wang YR. Manikins for textile evaluation-11 manikins for evaluation of pressure performance. UK: Woodhead Publishing, 2018, pp.241-258.

31. Liu K, Zeng X, Bruniaux P, et al. 3D interactive garment pattern-making technology. Comput Aid Des 2018; 104: 113-124.
32. Ashdown SP and DeLong M. Perception testing of apparel ease variation. Appl Ergon 1995; 26: 47-54.

33. Ashdown SP, Loker S, et al. Using 3D scans for fit analysis. J Text Apparel Technol Manag 2004; 4: 1-12.

34. Yu W. Subjective assessment of clothing fit. Clothing appearance and fit: science and technology. UK: Woodhead Publishing, 2004, pp.31-42.

35. Kim DE and LaBat K. An exploratory study of users' evaluations of the accuracy and fidelity of a three-dimensional garment simulation. Text Res J 2013; 83: 171-184.

36. Geršak J. Wearing comfort using body motion analysis. Anthropometry, apparel sizing and design. UK: Woodhead Publishing, 2014, pp.320-333.

37. Briceno L and Paul G. MakeHuman: a review of the modelling framework. In: proceedings of the 20th congress of the International Ergonomics Association (IEA 2018) (ed. Gianmaria Concheri), Venice, Italy, 1517 June 2011, pp. 224-232. Venice: Università degli Studi di Padova.

38. Tao F, Cheng J, Qi Q, et al. Digital replica-driven product design, manufacturing and service with big data. Int $J$ Adv Manuf Technol 2018; 94: 3563-3576.

39. Boschert $\mathrm{S}$ and Rosen R. Digital twin-the simulation aspect. Mechatron Future 2016; 7: 59-74.

40. Schleich B, Anwer N, et al. Shaping the digital replica for design and production engineering. CIRP Ann 2017; 66: 141-144.

41. Hunter L and Fan J. Fabric properties related to clothing appearance. Clothing appearance and fit: science and technology. UK: Woodhead Publishing, 2004, pp.89-111.

42. Ferguson-Pell M, Hagisawa $\mathrm{S}$, et al. Evaluation of a sensor for low interface pressure applications. Med Eng Phys 2000; 22: 657-663.

43. Li L, Au I, et al. A novel design method for an intelligent clothing based on garment design and knitting technology. Text Res J 2009; 79: 1670-1679.

44. Sun W, Zuo F, et al. Application of least square curve fitting algorithm based on LabVIEW in pressure detection system. In: 2015 international conference on applied science and engineering innovation, (ed. Xu M, Wang GL), Jinan, China, 30-31 August 2015, pp. pp. 39-43. Amsterdam: Advances in Engineering Research, Atlantis Press.

45. Hakala T, Puolakka A, et al. Application of air bladders for medical compression hosieries. Text Res $J$ 2018; 88: 2169-2181.

46. Makabe H, Momota H, et al. Effect of covered area at the waist on clothing pressure. Sen'i Gakkaishi 1993; 49: 513-521.

47. Varghese $\mathrm{N}$ and Thilagavathi G. Handle, fit and pressure comfort of silk/hybrid yarn woven stretch fabrics. Fiber Polym 2016; 17: 484-494.

48. Tanaka D, Yoshida M, et al. Effect of the peripheral pressure at groin region immediately after the wear of girdle on the rate of blood flow in the skin at the bottom of feet and surface humidity on the skin. $J$ Jpn Res Assoc Text End Use 1999; 40: 46-53. 
49. Erfan J. Study on the fit of young male pants. J Xi'an Univ Technol 2013; 1: 51-62.

50. Jin Z. Effect of seamless underwear on static pressure comfort of men under lower body. J Text Res 2009; 30: 99-103.

51. Liu R, Liu J, et al. Determination of leg cross-sectional curvatures and application in pressure prediction for lower body compression garments. Text Res $J$ 2019; 89: 1835-1852.

52. Rombaldoni F, Montarsolo A and Mazzuchetti G. KES-F characterization and hand evaluation of oxygen plasma-treated wool fabrics dyed at temperature below the boil. Text Res J 2010; 80: 1412-1421.

53. Zhong Y. Redressing three-dimensional garments based on pose duplication. Text Res $J$ 2010; 80: 904-916.

54. Lei G. Based on male physiological characteristics of the underwear structure comfort study. Shanghai Univ Eng Sci 2012; 12: 69-77.

55. Yu W. Clothing appearance and fit: science and technology-subjective assessment of clothing fit. UK: Woodhead Publishing, 2004, pp.31-42. 